\title{
TOWER SETS AND OTHER CONFIGURATIONS WITH THE COHEN-MACAULAY PROPERTY
}

\author{
GIUSEPPE FAVACCHIO, ALFIO RAGUSA AND GIUSEPPE ZAPPALÀ
}

\begin{abstract}
Some well-known arithmetically Cohen-Macaulay configurations of linear varieties in $\mathbb{P}^{r}$ as $k$-configurations, partial intersections and star configurations are generalized by introducing tower schemes. Tower schemes are reduced schemes that are finite union of linear varieties whose support set is a suitable finite subset of $\mathbb{Z}_{+}^{c}$ called tower set. We prove that the tower schemes are arithmetically Cohen-Macaulay and we compute their Hilbert function in terms of their support. Afterwards, since even in codimension 2 not every arithmetically CohenMacaulay squarefree monomial ideal is the ideal of a tower scheme, we slightly extend this notion by defining generalized tower schemes (in codimension 2) and we show that the support of these configurations (the generalized tower set) gives a combinatorial characterization of the primary decomposition of the arithmetically Cohen-Macaulay squarefree monomial ideals.
\end{abstract}

\section{INTRODUCTION}

In the last few years a large number of researchers in algebraic geometry in order to produce projective schemes with suitable Hilbert functions and graded Betti numbers constructed special configurations of linear varieties related to some subsets of $\mathbb{Z}_{+}^{c}$. Among these should be cited the partial intersection schemes introduced first in $[\mathrm{MR}]$ and generalized in any codimension in [RZ] and the $k$-configurations defined in [GS] and [GHS] to obtain maximal graded Betti numbers with respect to a fixed Hilbert function. On the other hand, to study the extremal Hilbert functions for fat point schemes in the plane, secant varieties of some classical algebraic varieties and some properties of the symbolic powers of ideals, the star configurations were defined and deeply investigated (see for instance [AS], GHM]). All these configurations lead to aCM ideals, mostly monomial and squarefree. Looking at what all these configurations have in common, in this paper we define the tower sets (Definition 2.1), suitable finite subsets of $\mathbb{Z}_{+}^{c}$, on which are supported the tower schemes (Definition 2.3), which generalize all the previous mentioned configurations. These tower sets enclose the combinatorial aspects of such configurations.

Also for these schemes we are able to prove that they have the aCM property (Theorem 2.6). Moreover, we compute the Hilbert function of the tower schemes in terms of its tower set support. At this point one can believe that, at least for monomial squarefree ideals, all aCM ideals can be constructed in this way. Unfortunately, already in codimension 2,

2010 Mathematics Subject Classification. 13 H 10, 14 N 20, 13 D 40.

Key words and phrases. Cohen-Macaulay, Monomial ideals, Configurations. 
this is false as we show in Example 3.4. So the question which arises is to find the right configuration which could characterize all the aCM monomial squarefree ideals in a polynomial ring. Here we give a complete answer in codimension 2 (Theorems 3.19, 3.32 and 3.35) defining a slight modification of the tower schemes (generalized tower sets and schemes, see Definitions 3.12 and 3.13). The codimension bigger than 2 case remains open.

After preliminaries and basic facts, in section 2 we introduce tower sets and tower schemes and we prove that all these schemes are aCM (Theorem 2.6). Then we show that every tower scheme has the same Hilbert function as a corresponding tower scheme supported on a left segment whose Hilbert function was computed in [RZ] (see Proposition 2.11 and Corollary 2.12). Section 3 is devoted to give a combinatorial characterization for aCM squarefree monomial ideals of codimension 2. To do that we give a slight generalization of tower sets and tower schemes (Definitions 3.12 and 3.13). Then we prove numerous preparatory results about these sets and schemes and finally in Theorems 3.19 and 3.35 we prove the stated characterization.

\section{Notation AND PRELIMINARIES}

Throughout the paper $k$ will be a field and $R:=k\left[x_{1}, \ldots, x_{n}\right]=\oplus_{d} R_{d}$ will be the standard graded polynomial $k$-algebra.

We will denote by $\mathbb{Z}_{+}:=\{r \in \mathbb{Z} \mid r>0\}$. If $r \in \mathbb{Z}_{+}$we will set $[r]:=$ $\{1, \ldots, r\}$. If $c, r \in \mathbb{Z}_{+}$we will denote by $C_{c, r}$ the set of the subsets of $[r]$ of cardinality $c$.

Moreover, we will set $\pi_{i}: \mathbb{Z}_{+}^{c} \rightarrow \mathbb{Z}_{+}$the projection on the $i$-th component. On the set $\mathbb{Z}_{+}^{c}$ we will use the following standard partial order. If $\alpha, \beta \in \mathbb{Z}_{+}^{c}$, $\alpha \leq \beta$ iff $\pi_{i}(\alpha) \leq \pi_{i}(\beta)$ for every $i \in[c]$.

We will denote by $\left(\mathbb{Z}_{+}^{c}\right)^{*}:=\left\{\left(a_{1}, \ldots, a_{c}\right) \in \mathbb{Z}_{+}^{c} \mid a_{i} \neq a_{j}\right.$ for every $\left.i \neq j\right\}$. Let $T \subset \mathbb{Z}_{+}^{c}$ be a finite set. Let $1 \leq t \leq c-1$ be an integer and let $\alpha \in \mathbb{Z}_{+}^{t}$. We set

$$
T_{\alpha}:=\left\{\gamma \in \mathbb{Z}_{+}^{c-t} \mid(\gamma, \alpha) \in T\right\}
$$

and

$$
T^{\alpha}:=\left\{\gamma \in \mathbb{Z}_{+}^{c-t} \mid(\alpha, \gamma) \in T\right\}
$$

Definition 1.1. The function $\varphi:\left(\mathbb{Z}_{+}^{c}\right)^{*} \rightarrow C_{c, n}$ such that $\varphi\left(a_{1}, \ldots, a_{c}\right)=$ $\left\{a_{1}, \ldots, a_{c}\right\}$, will be called forgetful function. A function $\omega: C_{c, n} \rightarrow\left(\mathbb{Z}_{+}^{c}\right)^{*}$ will be called ordinante iff $\varphi \circ \omega=\operatorname{id}_{C_{c, n}}$.

Let $L \subset \mathbb{Z}_{+}^{c}$ be a finite set. $L$ is said left segment if for every $\alpha \in L$ and $\beta \in \mathbb{Z}_{+}^{c}$ with $\beta \leq \alpha$ it follows that $\beta \in L$.

Let $L \subset \mathbb{Z}_{+}^{c}$ be a left segment. The set $\left\{\alpha_{1}, \ldots, \alpha_{r}\right\} \subseteq L$ is called set of generators for $L$ if for every $\alpha \in L, \alpha \leq \alpha_{i}$ for some $i$. The element $\left(\max \pi_{1}(L), \ldots, \max \pi_{c}(L)\right) \in \mathbb{Z}_{+}^{c}$ is said the size of $L$.

Let $L \subset \mathbb{Z}_{+}^{c}$ be a left segment of size $\left(m_{1}, \ldots, m_{c}\right)$, with $c<n$. For $1 \leq i \leq c$, let $\mathcal{F}_{i}=\left\{f_{i 1}, \ldots, f_{i m_{i}}\right\}$ be $c$ families of generic linear forms belonging to $R$. For every $\alpha=\left(a_{1}, \ldots, a_{c}\right) \in L$ we set $I_{\alpha}:=\left(f_{1 a_{1}}, \ldots, f_{c a_{c}}\right)$. We recall that the scheme defined by the ideal $I_{L}\left(\mathcal{F}_{1}, \ldots, \mathcal{F}_{c}\right):=\bigcap_{\alpha \in L} I_{\alpha}$ is called partial intersection, with support on $L$ and with respect to the families $\mathcal{F}_{1}, \ldots, \mathcal{F}_{c}$. 
If $\alpha \in \mathbb{Z}_{+}^{c}$ we set $v(\alpha):=\sum_{i=1}^{c} \pi_{i}(\alpha)$. If $L \subset \mathbb{Z}_{+}^{c}$ is a left segment, the Hilbert function of $L$ is

$$
H_{L}(i):=|\{\alpha \in L \mid v(\alpha)=i+c\}| .
$$

We remind that $H_{L}$ coincides with the Hilbert function of a partial intersection supported on $L$ (for details about left segments and partial intersections see [RZ]).

In the sequel, if $M$ is a matrix of rank $r$, with entries in $R$, we will denote by $I(M)$ the ideal generated by the minors of size $r$ in $M$.

\section{TOWERS SETS}

Many recent papers dealt with special configurations of linear subvarieties of projective spaces which raised up to Cohen-Macaulay varieties, for instance partial intersections studied in [RZ], $k$-configurations studied in [GHS, star configurations studied in GHM]. In this section we would like to generalize all these configurations in such a way to preserve the CohenMacaulayness.

Definition 2.1. Let $T \subset \mathbb{Z}_{+}^{c}$ be a finite set. We say that $T$ is a tower set if for every $t \in[c-1]$ and for every $\alpha, \beta \in \mathbb{Z}_{+}^{t}$, with $\alpha<\beta, T_{\alpha} \neq \emptyset$, we have $T_{\alpha} \supseteq T_{\beta}$.

Note that when $c=1$ every finite subset of $\mathbb{Z}_{+}$is a tower set.

Remark 2.2. If $T \subset \mathbb{Z}_{+}^{c}$ is a tower set and $\alpha \in \mathbb{Z}_{+}^{t}$ then $T_{\alpha} \subset \mathbb{Z}_{+}^{c-t}$ is also a tower set.

Definition 2.3. Let $T \subset \mathbb{Z}_{+}^{c}$ be a tower set. Let $R:=k\left[x_{1}, \ldots, x_{n}\right]$, with $2 \leq c \leq n-1$. Let $\mathcal{F}_{i}=\left\{f_{i j} \mid j \in \pi_{i}(T)\right\}, 1 \leq i \leq c$, where $f_{i j} \in R_{d_{i j}}$, such that $f_{i j}$ and $f_{i h}$ are coprime when $j \neq h$ and for every $\alpha=\left(a_{1}, \ldots, a_{c}\right) \in T$ the sequence $\left(f_{1 a_{1}}, \ldots, f_{c a_{c}}\right)$ is regular. We will denote by $I_{\alpha}$ the complete intersection ideal generated by $f_{1 a_{1}}, \ldots, f_{c a_{c}}$. We set

$$
I_{T}\left(\mathcal{F}_{1}, \ldots, \mathcal{F}_{c}\right):=\bigcap_{\alpha \in T} I_{\alpha}
$$

It defines a c-codimensional subscheme of $\mathbb{P}^{n}$ called tower scheme, with support on $T$, with respect to the families $\mathcal{F}_{1}, \ldots, \mathcal{F}_{c}$.

Note that if $T$ is a $c$-left segment then $T$ is a tower set, so every partial intersection is a tower scheme.

Recently many people investigated special subschemes called star configurations. We recall that a star configuration is defined as follows. Let $R:=k\left[x_{1}, \ldots, x_{n}\right], s, c \in \mathbb{Z}_{+}$such that $c \leq \min \{s, n-1\}$. Take a set $\mathcal{F}$ consisting of $s$ forms $f_{1}, \ldots, f_{s} \in R$ such that any $c$ of them are a regular sequence. If $s \geq a_{1}>\ldots>a_{c} \geq 1$ are integers and $\alpha=\left\{a_{1}, \ldots, a_{c}\right\}$ we set $I_{\alpha}:=\left(f_{a_{1}}, \ldots, f_{a_{c}}\right)$. A star configuration is the subscheme $V_{c}\left(\mathcal{F}, \mathbb{P}^{n}\right) \subset \mathbb{P}^{n}$ defined by the ideal $\bigcap I_{\alpha}$ where $\alpha$ runs over all the subsets of $[s]$ of cardinality c. For more details on star configurations see, for instance, GHM].

Remark 2.4. A star configuration is a particular tower scheme. Namely, let

$$
T=\left\{\left(a_{1}, \ldots, a_{c}\right) \in \mathbb{Z}_{+}^{c} \mid s \geq a_{1}>\ldots>a_{c} \geq 1\right\},
$$


and let us consider the families of forms

$$
\mathcal{F}_{i}=\left(f_{s-i+1}, \ldots, f_{c-i+1}\right), \quad \text { for } 1 \leq i \leq c .
$$

$T$ is trivially a tower set and $V\left(I_{T}\left(\mathcal{F}_{1}, \ldots, \mathcal{F}_{c}\right)\right)=V_{c}\left(\mathcal{F}, \mathbb{P}^{n}\right)$.

In the quoted papers it was shown that partial intersections, $k$-configurations and star configurations are all aCM schemes. Now we prove that every tower scheme is an aCM scheme and this generalizes those results. We need the following lemma, which is a slight generalization of Lemma 1.6 in [RZ].

Lemma 2.5. Let $c, r \geq 2$ be integers. Let $V_{1} \supseteq \ldots \supseteq V_{r}$ be $(c-1)$ codimensional aCM subschemes of $\mathbb{P}^{n}$ and $A_{j}=V\left(f_{j}\right)$ hypersurfaces, $1 \leq$ $j \leq r$, with $\operatorname{deg} f_{j}=d_{j}$. We set $Y_{i}:=V_{i} \cap A_{i}$ and let us suppose that $Y_{i}$ is c-codimensional for each $i$ and that $Y_{i}$ and $Y_{j}$ have no common components for $i \neq j$. We set $d:=\sum_{i=1}^{r} d_{i}, Y:=Y_{1} \cup \ldots \cup Y_{r-1}$ and $X:=Y \cup Y_{r}$. Then the following sequence of graded $R$-modules

$$
0 \rightarrow I_{Y_{r}}\left(-\left(d-d_{r}\right)\right) \stackrel{f}{\rightarrow} I_{X} \stackrel{\varphi}{\rightarrow} I_{Y} /(f) \rightarrow 0
$$

is exact, where $f=\prod_{i=1}^{r-1} f_{i}$ and $\varphi$ is the natural map. Moreover

$$
I_{X}=I_{V_{1}}+f_{1} I_{V_{2}}+f_{1} f_{2} I_{V_{3}}+\cdots+f_{1} \ldots f_{r-1} I_{V_{r}}+\left(f_{1} \ldots f_{r}\right) .
$$

Proof. The proof is analogous to that of Lemma 1.6 in [RZ]. We report it for convenience of the reader.

Observe that the exactness of the above sequence in the middle depends on the fact that $f$ is regular modulo $I_{Y_{r}}$, since $Y_{i}$ and $Y_{j}$ have no common components for $i \neq j$. So, the only not trivial fact to prove is that the map $\varphi$ is surjective. For this we use induction on $r$. For $r=2$, since $V_{1}$ is aCM, $I_{Y}=I_{V_{1}}+\left(f_{1}\right)$, therefore every element in $I_{Y} /\left(f_{1}\right)$ looks like $z+\left(f_{1}\right)$ with $z \in I_{V_{1}} \subseteq I_{V_{2}}$. Hence, $z \in I_{Y_{1}} \cap I_{Y_{2}}=I_{X}$. So, $\varphi$ is surjective and the sequence is exact. Now, from the exactness of this sequence it follows that $I_{X}$ is generated by $I_{V_{1}}$ and $f_{1} I_{Y_{2}}$, i.e. $I_{X}=I_{V_{1}}+f_{1} I_{V_{2}}+\left(f_{1} f_{2}\right)$.

Let us suppose the lemma true for $r-1$. This means, in particular, that $I_{Y}=I_{V_{1}}+f_{1} I_{V_{2}}+\cdots+f_{1} \ldots f_{r-2} I_{V_{r-1}}+\left(f_{1} \ldots f_{r-1}\right)$. Therefore, every element $z \in I_{Y} /\left(f_{1} \ldots f_{r-1}\right)$ has the form $x+\left(f_{1} \ldots f_{r-1}\right)$ with $x \in I_{V_{1}}+$ $f_{1} I_{V_{2}}+\cdots+f_{1} \ldots f_{r-2} I_{V_{r-1}}$. Hence, $x \in I_{V_{r}}$ which implies $x \in I_{Y} \cap I_{Y_{r}}=I_{X}$. Again, by the exactness of our sequence we get that $I_{X}$ is generated by $f_{1} \ldots f_{r-1} I_{Y_{r}}$ and by $I_{V_{1}}+f_{1} I_{V_{2}}+\cdots+f_{1} \ldots f_{r-2} I_{V_{r-1}}$, i.e. $I_{X}=I_{V_{1}}+$ $f_{1} I_{V_{2}}+\cdots+f_{1} \ldots f_{r-1} I_{V_{r}}+\left(f_{1} \ldots f_{r}\right)$.

We are ready to prove our result.

Theorem 2.6. Every tower scheme is aCM.

Proof. Let $X$ be a tower scheme of codimension $c$. To show that $X$ is aCM we use induction on $c$. The property is trivially true for $c=1$, so we can assume that every tower scheme of codimension $c-1$ is aCM. Let $T$ be the support of $X$ and let $\mathcal{F}_{i}=\left\{f_{i j} \mid j \in \pi_{i}(T)\right\}$, for $1 \leq i \leq c$, be the families defining $X$. Let $\pi_{c}(T)=\left\{a_{1}, \ldots, a_{s}\right\}$, with $a_{1}<\ldots<a_{s}$. For every $i \in[s]$ we denote by $V_{i}$ the tower scheme of codimension $c-1$ supported on $T_{a_{i}}$. If $i<j$ then $V_{i} \supseteq V_{j}$. By inductive hypotheses each $V_{i}$ is aCM. Moreover we 
denote by $A_{i}$ the hypersurface defined by $f_{c a_{i}}$, with $i \in[s]$ and $Y_{i}=V_{i} \cap A_{i}$. Note that by the hypotheses on $\mathcal{F}_{h}$ 's $Y_{i}$ is aCM of codimension $c$.

Therefore we have $X=\bigcup_{1 \leq i \leq s} Y_{i}$. Now we use induction on $s$. For $s=1$ $X=Y_{1}$ is aCM. Suppose that $Y=\bigcup_{1 \leq i \leq s-1} Y_{i}$ is aCM and show that $X=$ $Y \cup Y_{s}$ is aCM. Applying the previous lemma we get the exact sequence

$$
0 \rightarrow I_{Y_{s}}(-\operatorname{deg} f) \rightarrow I_{X} \rightarrow I_{Y} /(f) \rightarrow 0
$$

where $f=\prod_{i=1}^{s-1} f_{c a_{i}}$ from which we see that a resolution of $I_{X}$ can be obtained as direct sum of the resolutions of $I_{Y_{s}}(-\operatorname{deg} f)$ and $I_{Y} /(f)$; since both have resolutions of length $c$ the same is true for $I_{X}$ and we are done.

Our next aim is to compute the Hilbert function of a tower scheme in the case when the defining families consist of linear forms. To do this, if $T$ is a tower set, we define a map $\sigma: T \rightarrow \mathbb{Z}_{+}^{c}$ as follows. Let $\alpha=\left(a_{1}, \ldots, a_{c}\right) \in T$, we set

$$
\begin{gathered}
h_{1}(\alpha):=\left|\left\{i \mid i \leq a_{1},\left(i, a_{2}, \ldots, a_{c}\right) \in T\right\}\right|, \\
h_{j}(\alpha):=\left|\left\{i \mid i \leq a_{j}, T_{\left(i, a_{j+1}, \ldots, a_{c}\right)} \neq \emptyset\right\}\right|, \text { for } 2 \leq j \leq c
\end{gathered}
$$

and finally

$$
\sigma\left(a_{1}, \ldots, a_{c}\right):=\left(h_{1}(\alpha), \ldots, h_{c}(\alpha)\right) .
$$

The map $\sigma$ is trivially injective. We set $T^{\#}:=\sigma(T)$.

Proposition 2.7. For every tower set $T, T^{\#}$ is a left segment.

Proof. Let $\alpha^{\prime}=\left(a_{1}^{\prime}, \ldots, a_{c}^{\prime}\right) \in T^{\#}$ and $\beta^{\prime}=\left(b_{1}^{\prime}, \ldots, b_{c}^{\prime}\right) \in \mathbb{Z}_{+}^{c}$, such that $\beta^{\prime} \leq \alpha^{\prime}$. We have to prove that $\beta^{\prime} \in T^{\#}$, i.e. we have to find $\beta \in T$ such that $\sigma(\beta)=\beta^{\prime}$. Let $\alpha=\left(a_{1}, \ldots, a_{c}\right) \in T$ be such that $\sigma(\alpha)=\alpha^{\prime}$, hence $a_{i}^{\prime}=h_{i}(\alpha)$. Since $b_{c}^{\prime} \leq h_{c}(\alpha)$, there is a unique element $b_{c}$ such that $T_{b_{c}} \neq \emptyset$ and $\left|\left\{i \mid i \leq b_{c}, T_{i} \neq \emptyset\right\}\right|=b_{c}^{\prime}$. Now, since $b_{c-1}^{\prime} \leq h_{c-1}(\alpha)$, we have that $T_{\left(b_{c-1}^{\prime}, a_{c}\right)} \neq \emptyset$ and, since $T$ is a tower set, $T_{\left(b_{c-1}^{\prime}, b_{c}\right)} \neq \emptyset$, therefore there is a unique element $b_{c-1}$ such that $T_{\left(b_{c-1}, b_{c}\right)} \neq \emptyset$ and $\mid\left\{i \mid i \leq b_{c-1}, T_{\left(i, b_{c}\right)} \neq\right.$ $\emptyset\} \mid=b_{c-1}^{\prime}$. By iterating the same argument we will set $b_{j}$ the unique element such that $T_{\left(b_{j}, b_{j+1}, \ldots, b_{c}\right)} \neq \emptyset$ and $\left|\left\{i \mid i \leq b_{j}, T_{\left(i, b_{j+1}, \ldots, b_{c}\right)} \neq \emptyset\right\}\right|=b_{j}^{\prime}$, for $1 \leq j \leq c$. Now we set $\beta=\left(b_{1}, \ldots, b_{c}\right)$. By definition $\beta \in T$ and $\sigma(\beta)=\beta^{\prime}$.

Remark 2.8. Note that if $T, U \subset \mathbb{Z}_{+}^{c}$ are tower sets such that $T \subseteq U$ then $T^{\#} \subseteq U^{\#}$.

Proposition 2.9. Let $T \subset \mathbb{Z}_{+}^{c}$ be a tower set. Let $X=V\left(I_{T}\left(\mathcal{F}_{1}, \ldots, \mathcal{F}_{c}\right)\right)$. Let $Y$ be a tower scheme supported on $T^{\#}$, with respect to the same families $\mathcal{F}_{1}, \ldots, \mathcal{F}_{c}$. Then $H_{X}=H_{Y}$.

Proof. If $c=1$ then $T$ is a finite subset of $\mathbb{Z}$, say $r=|T|$, so $T^{\#}=[r]$. Therefore $I_{X}$ and $I_{Y}$ are principal ideals generated by a form of same degree, hence $H_{X}=H_{Y}$.

So we may assume that $c \geq 2$ and we proceed by induction on $c$. We consider the set $\pi_{c}(T)=\left\{m_{1}, \ldots, m_{s}\right\}, m_{1}<\ldots<m_{s}$. Since $T$ is a tower set, $T_{m_{1}} \supseteq \ldots \supseteq T_{m_{s}}$ are $(c-1)$-tower sets. Let $X_{i}$ be the scheme defined by 
$I_{T_{m_{i}}}\left(\mathcal{F}_{1}, \ldots, \mathcal{F}_{c-1}\right)$. Then each $X_{i}$ is an aCM scheme of codimension $c-1$ by Theorem 2.6 and $X_{1} \supseteq \ldots \supseteq X_{s}$. Moreover, by Remark 2.8. $\left(T_{m_{1}}\right)^{\#} \supseteq$ $\ldots \supseteq\left(T_{m_{s}}\right) \#$. Let $Y_{i}$ be the scheme defined by $I_{\left(T_{m_{i}}\right)}\left(\mathcal{F}_{1}, \ldots, \mathcal{F}_{c-1}\right)$. By the inductive hypothesis $H_{X_{i}}=H_{Y_{i}}$. Now, let $\mathcal{F}_{c}=\left\{f_{1}, \ldots, f_{s}\right\}$, we set $\bar{X}_{i}:=X_{i} \cap V\left(f_{i}\right)$ and $\bar{Y}_{i}=Y_{i} \cap V\left(f_{i}\right)$. Since $X_{i}$ and $Y_{i}$ are aCM then $H_{\bar{X}_{i}}=H_{\bar{Y}_{i}}$. Finally, using induction on $s$ and the exact sequences (see Lemma 2.5)

$$
\begin{aligned}
0 & \rightarrow I_{\bar{X}_{s}}(-\delta) \rightarrow I_{X} \rightarrow I_{\bar{X}_{i} \cup \ldots \cup \bar{X}_{s-1}} /\left(f_{1} \ldots f_{s-1}\right) \rightarrow 0 \\
0 & \rightarrow I_{\bar{Y}_{s}}(-\delta) \rightarrow I_{Y} \rightarrow I_{\bar{Y}_{i} \cup \ldots \cup \bar{Y}_{s-1}} /\left(f_{1} \ldots f_{s-1}\right) \rightarrow 0
\end{aligned}
$$

where $\delta=\operatorname{deg}\left(f_{1} \ldots f_{s-1}\right)$, we get the conclusion.

Proposition 2.9 allows us to find a formula for the Hilbert function of a tower scheme.

Remark 2.10. Note that, according to the exact sequence of Lemma 2.5. the Hilbert function of a tower scheme depends on the tower set and on the degrees of the forms in the families.

Now we associate to a tower scheme $X$, supported on a left segment $L$, a partial intersection $Y$ with support on a suitable left segment $L_{D}$ such that $H_{X}=H_{Y}$.

If $L$ is a left segment of size $\left(a_{1}, \ldots, a_{c}\right)$ and $D=\left\{d_{i j}\right\}, 1 \leq i \leq c$ and $1 \leq j \leq a_{i}$ are positive integers, we define a new left segment, which we will be denoted by $L_{D}$, in the following way. If $L$ is (minimally) generated by $K_{1}, \ldots, K_{r}$, then $L_{D}$ is the left segment generated by $K_{1}^{\prime}, \ldots, K_{r}^{\prime}$ where, if $K_{i}=\left(k_{1}, \ldots, k_{c}\right)$ then $K_{i}^{\prime}=\left(\sum_{j=1}^{k_{1}} d_{1 j}, \ldots, \sum_{j=1}^{k_{c}} d_{c j}\right)$.

Thus, let $X$ be a tower scheme supported on the left segment $L$ and let $\mathcal{F}_{i}=\left\{f_{i j} \mid j \in \pi_{i}(L)\right\}$, for $1 \leq i \leq c$, be the families defining $X$. Set now $\pi_{c}(L)=\left\{a_{1}, \ldots, a_{c}\right\}$, with $a_{1}<\ldots<a_{c}$ and $D=\left\{d_{i j}\right\}$ where $d_{i j}=\operatorname{deg} f_{i j}$, $1 \leq i \leq c$ and $1 \leq j \leq a_{i}$. Since, by Remark 2.10, $H_{X}$ depends just on $D$ we may assume that $f_{i j}=\prod_{h=1}^{d_{i j}} l_{i j}^{h}$, where each $l_{i j}^{h}$ is a linear form. Now we denote by $Y$ the partial intersection supported on $L_{D}$ with respect the $c$ ordered families of linear forms

$$
\mathcal{L}_{i}=\left(l_{i 11}, \ldots, l_{i 1 d_{i 1}}, l_{i 21}, \ldots, l_{i 2 d_{i 2}}, \ldots, l_{i a_{i} 1}, \ldots, l_{i a_{i} d_{i a_{i}}}\right) .
$$

Proposition 2.11. Given a tower scheme $X$ supported on the left segment $L$ with respect to the families of forms $\mathcal{F}_{i}=\left\{f_{i j} \mid j \in \pi_{i}(L)\right\}$, for $1 \leq i \leq c$ and $D=\left\{d_{i j}\right\}$ where $d_{i j}=\operatorname{deg} f_{i j}$. Then $H_{X}=H_{L_{D}}$.

Proof. By definition $I_{X}=\cap_{\left(j_{1}, \ldots, j_{c}\right) \in L}\left(f_{1 j_{1}}, \ldots, f_{c j_{c}}\right)$. Now we denote by $Y$ the partial intersection supported on $L_{D}$ with respect the $c$ ordered families of linear forms $\mathcal{L}_{i}=\left(l_{i 11}, \ldots, l_{i 1 d_{i 1}}, l_{i 21}, \ldots, l_{i 2 d_{i 2}}, \ldots, l_{i a_{i} 1}, \ldots, l_{i a_{i} d_{i a_{i}}}\right), 1 \leq$ $i \leq c$. Now if $\alpha$ is an integer such that $1 \leq \alpha \leq \sum_{s=1}^{a_{i}} d_{i s}$ we set

$$
t_{\alpha}:=\max \left\{j \mid d_{i 1}+\ldots+d_{i j}<\alpha\right\}+1
$$

and

$$
h_{\alpha}:=\alpha-\sum_{s=1}^{t_{\alpha}-1} d_{i s}
$$


and $p_{i \alpha}:=l_{i t_{\alpha} h_{\alpha}}$. Then, with this notation

$$
I_{Y}=\bigcap_{\left(\alpha_{1}, \ldots, \alpha_{c}\right) \in L_{D}}\left(p_{1 \alpha_{1}}, \ldots, p_{c \alpha_{c}}\right) .
$$

It is a matter of computation to show that $I_{X}=I_{Y}$ and this completes the proof.

In the next corollary we lead back the computation of the Hilbert function of a tower scheme to that of a partial intersection. The Hilbert function of a partial intersection was explicitly computed in [RZ].

Corollary 2.12. If $X$ is a tower scheme supported on a tower set $T$ with respect to families of forms of degrees $D$, then $H_{X}=H_{\left(T^{\#}\right)_{D}}$.

Proof. It follows just using Propositions 2.9 and 2.11 .

\section{Generalized tower sets: a characterization of aCM PROPERTY}

In this section we will generalize tower sets in such a way to characterize aCM squarefree monomial ideal of codimension 2 .

Let $I \subset k\left[x_{1}, \ldots, x_{n}\right]$ be an equidimensional squarefree monomial ideal of codimension $c$ and let $I=\mathfrak{p}_{1} \cap \ldots \cap \mathfrak{p}_{t}$ be its primary decomposition. Each $\mathfrak{p}_{i}$ is a prime ideal of the type $\left(x_{a_{i 1}}, \ldots, x_{a_{i c}}\right)$. So we can consider the subset $\mathcal{S}(I):=\left\{\left\{a_{i 1}, \ldots, a_{i c}\right\} \mid 1 \leq i \leq t\right\}$ of $C_{c, n}$.

Vice versa to $\mathcal{S} \subseteq C_{c, n}$ we can associate an equidimensional squarefree monomial ideal

$$
I_{\mathcal{S}}:=\bigcap_{\left\{a_{1}, \ldots, a_{c}\right\} \in \mathcal{S}}\left(x_{a_{1}}, \ldots, x_{a_{c}}\right) .
$$

Definition 3.1. Let $\mathcal{S} \subseteq C_{c, n}$. We will say $\mathcal{S}$ aCM if $I_{\mathcal{S}}$ is an aCM ideal.

Definition 3.2. Let $\mathcal{S} \subseteq C_{c, n}$. We will say that $\mathcal{S}$ is towerizable if there exists a permutation $\tau$ on $[n]$ and an ordinante function $\omega: C_{c, n} \rightarrow\left(\mathbb{Z}_{+}^{c}\right)^{*}$ such that $\tau(\omega(\mathcal{S}))$ is a tower set.

Remark 3.3. Let $\mathcal{S} \subseteq C_{c, n}$. Note that $\mathcal{S}$ is towerizable if there exists a tower set $T$ and families $\mathcal{F}_{i} \subseteq\left\{x_{1}, \ldots, x_{n}\right\}$, such that $\mathcal{S}\left(I_{T}\left(\mathcal{F}_{1}, \ldots, \mathcal{F}_{c}\right)\right)=\mathcal{S}$.

By Theorem 2.6 if $\mathcal{S}$ is towerizable then $\mathcal{S}$ is aCM, however there are aCM equidimensional squarefree monomial ideals $I$ such that $\mathcal{S}(I)$ is not towerizable. Here it is an example in codimension 2.

Example 3.4. Let $\mathcal{S}=\{\{1,2\},\{3,4\},\{5,6\},\{4,6\},\{1,4\},\{1,6\}\}$. Then it is easy to check that $I_{\mathcal{S}}$ is the determinantal ideal generated by the order 3 minors of the matrix

$$
\left(\begin{array}{ccc}
x_{1} & 0 & 0 \\
x_{2} & x_{3} & x_{5} \\
0 & x_{4} & 0 \\
0 & 0 & x_{6}
\end{array}\right),
$$

so $\mathcal{S}$ is aCM. Let us suppose that $\mathcal{S}$ is towerizable. Then there exists a tower scheme $X$ with support on a tower set $T$ such that $\mathcal{S}\left(I_{X}\right)=\mathcal{S}$. Of course $|T|=6$ and there is not a variable $x_{k}$ such that the ideal $\left(x_{k}\right)$ contains 4 of the 6 minimal primes of $I_{\mathcal{S}}$. Consequently, $\left|\pi_{2}(T)\right| \leq 3$ and for every $a \in \pi_{2}(T)\left|T_{a}\right| \leq 3$ so we have only three possibilities 
1) $\pi_{2}(T)=\{a, b\}$ with $\left|T_{a}\right|=3$ and $\left|T_{b}\right|=3$;

2) $\pi_{2}(T)=\{a, b, c\}$ with $\left|T_{a}\right|=2,\left|T_{b}\right|=2$ and $\left|T_{c}\right|=2$;

3) $\pi_{2}(T)=\{a, b, c\}$ with $\left|T_{a}\right|=3,\left|T_{b}\right|=2$ and $\left|T_{c}\right|=1$.

The first two cases cannot occur since $I_{X}=I_{S}$ does not contain monomials of degree two.

Therefore $T_{a} \supset T_{b} \supset T_{c}$ and $T_{a}=\left\{h_{1}, h_{2}, h_{3}\right\}, T_{b}=\left\{h_{1}, h_{2}\right\}, T_{c}=\left\{h_{1}\right\}$ for some $h_{i}$ 's and thus $T=\left\{\left(h_{1}, a\right),\left(h_{2}, a\right),\left(h_{3}, a\right),\left(h_{1}, b\right),\left(h_{2}, b\right),\left(h_{1}, c\right)\right\}$. But the numbers 2, 3 and 5 belong each to one only element of $S$ whereas in $T$ there are only two such numbers, precisely $h_{3}$ and $c$.

Because of the previous example it is natural to ask which sets $\mathcal{S} \subseteq C_{c, n}$ are aCM. We will give a characterization in codimension 2 (see Theorems 3.19 and 3.35 .

Definition 3.5. Let $h \in \mathbb{Z}_{+}$. Let $\mathcal{S} \subseteq C_{2, n}$. We set

$$
\mathcal{S}: h:=\{A \in \mathcal{S} \mid h \notin A\} .
$$

If $S \subseteq \mathbb{Z}_{+}^{2}$ we set

$$
S: h:=\left\{\alpha \in S \mid \pi_{1}(\alpha) \neq h \text { and } \pi_{2}(\alpha) \neq h\right\} .
$$

Remark 3.6. Note that if $\mathcal{S}$ is aCM then $\mathcal{S}: h$ is aCM. Indeed, if $M$ is an Hilbert-Burch matrix for $I_{S}$ then $I_{\mathcal{S}: h}$ is generated by the maximal minors of the matrix obtained from $M$ by replacing $x_{h}$ with 1 .

In the sequel we will use the following result which shows that if $S \subseteq C_{2, n}$ is aCM then also the scheme obtained by replacing $\left(x_{i}, x_{j}\right) \supseteq I_{\mathcal{S}}$ with $\left(h_{i}, h_{j}\right)$, generic complete intersections, is aCM.

Proposition 3.7. Let $\mathcal{S} \subseteq C_{2, n}$ be an aCM set, with $I_{\mathcal{S}} \subset k\left[x_{1}, \ldots, x_{n}\right]$. Let $h_{1}, \ldots, h_{n} \in k\left[y_{1}, \ldots, y_{m}\right]$ be forms, such that $\operatorname{depth}\left(h_{i}, h_{j}\right)=2$ for every $\{i, j\} \in \mathcal{S}$ and

$$
\operatorname{depth}\left(h_{i}, h_{j}, h_{u}, h_{v}\right) \geq 3
$$

for every $\{i, j\},\{u, v\} \in \mathcal{S},\{i, j\} \neq\{u, v\}$. Then the ideal $J=\bigcap_{\{i, j\} \in \mathcal{S}}\left(h_{i}, h_{j}\right)$ is $a C M$.

Proof. We consider the following vectors

$$
\underline{x}=\left(x_{1}, \ldots, x_{n}\right) \text { and } \underline{h}=\left(h_{1}, \ldots, h_{n}\right) .
$$

Since $I_{\mathcal{S}}$ is aCM we can consider $M=M(\underline{x})$, an Hilbert-Burch matrix for $I_{\mathcal{S}}$. We claim that $N=M(\underline{h})$ is an Hilbert-Burch matrix for $J$. We have to prove that $J=I(N)$. Let $g \in I(N)$ be a maximal minor of $N$. Then $g=f(\underline{h})$, with $f(\underline{x}) \in I_{S}$. Therefore $f(\underline{x})=\lambda_{i}(\underline{x}) x_{i}+\mu_{j}(\underline{x}) x_{j}$ for every $\{i, j\} \in S$, consequently $f(\underline{h}) \in\left(h_{i}, h_{j}\right)$ for every $\{i, j\} \in \mathcal{S}$. So $I(N) \subseteq J$.

To conclude the proof it is enough to show that $\operatorname{deg} I(N)=\operatorname{deg} J$. By the generality of the forms $h_{1}, \ldots, h_{n}$, we have that

$$
\operatorname{deg} J=\sum_{\{i, j\} \in \mathcal{S}}\left(\operatorname{deg} h_{i}\right)\left(\operatorname{deg} h_{j}\right) .
$$

Now we proceed by induction on $n$. If $n=2$ then $\mathcal{S}=\{1,2\}$ and $I(N)=$ $\left(h_{1}, h_{2}\right)$. So we can suppose that $\operatorname{deg} I(N)=\sum_{\{i, j\} \in \mathcal{S}}\left(\operatorname{deg} h_{i}\right)\left(\operatorname{deg} h_{j}\right)$, when $\mathcal{S} \subseteq C_{2, n-1}$. We can write $\mathcal{S}=(\mathcal{S}: n) \cup \mathcal{S}_{(n)}$ where $\mathcal{S}: n=\{\alpha \in S \mid n \notin \alpha\}$ 
and $\mathcal{S}_{(n)}=\mathcal{S} \backslash(\mathcal{S}: n)$. By Remark [3.6, $\mathcal{S}: n$ is aCM. Let $\widehat{M}(\underline{x})$ be an Hilbert-Burch matrix for $I_{\mathcal{S}: n}$. We set $\widehat{N}=\widehat{M}(\underline{h})$. Therefore

$$
I_{\mathcal{S}}=I(M)=\left(I(M): x_{n}\right) \cap\left(x_{n}, \prod_{u \in \mathcal{S}_{(n)}} x_{u}\right)=I(\widehat{M}) \cap\left(x_{n}, \prod_{u \in \mathcal{S}_{(n)}} x_{u}\right) .
$$

Hence, using the inductive hypothesis, we get

$$
\begin{gathered}
\left.\operatorname{deg} I(N)=\operatorname{deg}(I(\widehat{N}))+\operatorname{deg}\left(h_{n}, \prod_{u \in \mathcal{S}_{(n)}} h_{u}\right)\right)= \\
\sum_{\{i, j\} \in(\mathcal{S}: n)}\left(\operatorname{deg} h_{i}\right)\left(\operatorname{deg} h_{j}\right)+\left(\operatorname{deg} h_{n}\right) \sum_{\{u, n\} \in \mathcal{S}_{(n)}} \operatorname{deg} h_{u}=\sum_{\{i, j\} \in \mathcal{S}}\left(\operatorname{deg} h_{i}\right)\left(\operatorname{deg} h_{j}\right) .
\end{gathered}
$$

We recall that if $T \subseteq \mathbb{Z}_{+}^{2}$ and $i \in \mathbb{Z}_{+}$then

$$
T^{i}=\left\{j \in \pi_{2}(T) \mid(i, j) \in T\right\}
$$

and

$$
T_{i}=\left\{j \in \pi_{1}(T) \mid(j, i) \in T\right\} .
$$

Remark 3.8. Let $T \subset\left(\mathbb{Z}_{+}^{2}\right)^{*}$ be a tower set. Then

1) $a<b$ and $T_{a} \neq \emptyset \Rightarrow(a, b) \notin T$. Indeed, the assumption implies $T_{a} \supseteq T_{b}$. Since $(a, a) \notin T$, we have $a \notin T_{a}$, therefore $a \notin T_{b}$ i.e. $(a, b) \notin T$.

2) $a<b$ and $(b, a) \in T \Rightarrow(a, b) \notin T$. Indeed, the assumption implies $T_{a} \neq \emptyset$ so, by the previous item, $(a, b) \notin T$.

3) $\{(a, b),(b, a)\} \nsubseteq T$ for every $a$ and $b$. It follows by item 2 .

Note that by item $3,|T|=|\varphi(T)|$ where $\varphi$ is the forgetful function.

Proposition 3.9. Let $T \subset \mathbb{Z}_{+}^{2}$ be a tower set. Then $T^{i}$ and $T^{h}$ are comparable under inclusion for every $i$ and $h$.

Proof. Let $j \in T^{i}$ be such that $j \notin T^{h}$, we have to show that $T^{h} \subset T^{i}$. Let $k \in T^{h}$, i.e. $h \in T_{k}$; but $h \notin T_{j}$ therefore since $T$ is a tower set we have that $T_{j} \subset T_{k}$, so $i \in T_{k}$ i.e. $(i, k) \in T$ that implies that $k \in T^{i}$.

Proposition 3.10. Let $T \subset\left(\mathbb{Z}_{+}^{2}\right)^{*}$ be a tower set.

1) Let $h \in \pi_{2}(T)$ be such that $T_{h} \supseteq T_{j}$ for every $j \in \pi_{2}(T)$. Then $h \notin \pi_{1}(T)$.

2) Let $h \in \pi_{1}(T)$ be such that $T^{h} \supseteq T^{i}$ for every $i \in \pi_{1}(T)$. Then $h \notin \pi_{2}(T)$.

Proof. 1) If $(h, j) \in T$ then $h \in T_{j} \subseteq T_{h}$, i.e. $(h, h) \in T$.

2) Using Proposition 3.9 the proof is analogous to item 1.

Let $T \subset\left(\mathbb{Z}_{+}^{2}\right)^{*}$ be a tower set. Let $h \in \pi_{1}(T) \cap \pi_{2}(T)$. We set

$$
F_{T}(h):=\left\{j \in \pi_{2}(T) \mid T_{h} \subset T_{j} \text { and }(h, j) \notin T\right\} .
$$

Note that if $j \in F_{T}(h)$ then $j<h$. 
Definition 3.11. Let $U \subseteq C_{2, n}$. We say that $U$ is connected if for every $A, B \in U$ there is $C \in U$ such that $A \cap C \neq \emptyset$ and $B \cap C \neq \emptyset$. Let $S \subset\left(\mathbb{Z}_{+}^{2}\right)^{*}$. We say that $S$ is connected if $\varphi(S)$ is connected.

Definition 3.12. Let $S \subset\left(\mathbb{Z}_{+}^{2}\right)^{*}$ be a finite set. We say that $S$ is a generalized tower set if

1) $S$ is connected;

2) $S=T \cup S_{0}$ where $T$ is a tower set

and $S_{0}$ has the following further properties

3) for every $(i, j) \in S_{0}, i \notin \pi_{1}(T) \cup \pi_{2}(T)$ and $j \in \pi_{1}(T) \cap \pi_{2}(T)$;

4) for every $(i, j) \in S_{0}$ and $h \in F_{T}(j),(i, h) \in S_{0}$.

Definition 3.13. Let $S \subset\left(\mathbb{Z}_{+}^{2}\right)^{*}$ be a generalized tower set. Let $R=$ $k\left[x_{1}, \ldots, x_{n}\right], n \geq 3$. Let $\mathcal{F}_{i}=\left\{f_{i j} \mid j \in \pi_{i}(S)\right\}, 1 \leq i \leq 2$, where each $f_{i j}$ is a form satisfying such conditions of genericity: for every $\left(a_{1}, a_{2}\right) \in S, f_{1 a_{1}}, f_{2 a_{2}}$ are coprime and for every $\left(a_{1}, a_{2}\right),\left(b_{1}, b_{2}\right) \in S$, with $\left\{a_{1}, a_{2}\right\} \neq\left\{b_{1}, b_{2}\right\}$, $\operatorname{depth}\left(f_{1 a_{1}}, f_{2 a_{2}}, f_{1 b_{1}}, f_{2 b_{2}}\right) \geq 3$. If $\alpha=\left(a_{1}, a_{2}\right) \in S$, we will denote by $I_{\alpha}$ the complete intersection ideal generated by $f_{1 a_{1}}, f_{2 a_{2}}$. We set

$$
I_{S}\left(\mathcal{F}_{1}, \mathcal{F}_{2}\right):=\bigcap_{\alpha \in S} I_{\alpha} .
$$

It defines a 2-codimensional subscheme of $\mathbb{P}^{n}$ called generalized tower scheme, with support on $S$, with respect to the families $\mathcal{F}_{1}, \mathcal{F}_{2}$.

In the sequel if $S \subset\left(\mathbb{Z}_{+}^{2}\right)^{*}$ we will set for short $I_{S}:=I_{\varphi(S)}$, consequently $S$ will be said aCM if $I_{S}$ is aCM.

In order to prove our results on the Cohen-Macaulayness of such schemes we need several lemmas.

Lemma 3.14. Let $S=T \cup S_{0}$ be a generalized tower set. Let $i \in \pi_{1}\left(S_{0}\right)$ and let $m=\min \left\{j \mid(i, j) \in S_{0}\right\}$. Then $F_{T}(m)=\emptyset$.

Proof. Let $s \in F_{T}(m)$; then $s<m$ and by Definition 3.12, item $4,(i, s) \in S_{0}$, which is a contradiction.

Lemma 3.15. With the above notation, if $h \in \pi_{1}(T) \cap \pi_{2}(T)$ then for every $j \in \pi_{1}(T) \cap \pi_{2}(T) \backslash\{h\}$ we have $F_{T: h}(j) \subseteq F_{T}(j)$.

Proof. If $b \in F_{T: h}(j)$ then $(T: h)_{j} \subset(T: h)_{b}$ and $(j, b) \notin T: h$, with $j \neq h$ and $b \neq h$, so $(j, b) \notin T$. Moreover there is $a$ such that $(a, b) \in T: h$ and $(a, j) \notin T: h$. Since $a \neq h$ this implies that $T_{b} \not \subset T_{j}$. Since $T$ is a tower set we get that $T_{j} \subset T_{b}$.

Lemma 3.16. Let $S=T \cup S_{0}$ be a generalized tower set. Let $h \in \pi_{1}(T) \cap$ $\pi_{2}(T)$. Then $S: h$ is a generalized tower set with respect to the decomposition $S: h=(T: h) \cup\left(S_{0}: h\right)$.

Proof. Of course $S: h=(T: h) \cup\left(S_{0}: h\right)$.

1) Since $S$ is connected then $S: h$ is connected too.

2) Let $a, b \in \pi_{2}(T: h), a<b$. Let $i \in(T: h)_{b}$; then $(i, b) \in T: h$ i.e. $i \in T_{b} \subseteq T_{a}$; since $i \neq h$ and $b \neq h$ then $i \in(T: h)_{a}$. 
3) Let $(i, j) \in S_{0}: h$. Of course $i \notin \pi_{1}(T: h) \cup \pi_{2}(T: h)$. Moreover $\left(\pi_{1}(T) \cap \pi_{2}(T)\right) \backslash\{h\}=\pi_{1}(T: h) \cap \pi_{2}(T: h)$. Indeed, $\pi_{1}(T: h) \cap$ $\pi_{2}(T: h) \subseteq\left(\pi_{1}(T) \cap \pi_{2}(T)\right) \backslash\{h\}$ trivially; if $k \in\left(\pi_{1}(T) \cap \pi_{2}(T)\right) \backslash\{h\}$ then $k \neq h, k \in T_{b}$ where $T_{b} \supseteq T_{u}$ for every $u$ and $k \in T^{a}$ where $T^{a} \supseteq T^{v}$ for every $v$, (see Proposition [3.9), so by Proposition 3.10, $a \neq h$ and $b \neq h$ i.e. $(a, k),(k, b) \in T: h$, hence item 3 is clear.

4) Let $(i, j) \in S_{0}: h$ and $u \in F_{T: h}(j)$. By Lemma 3.15, $u \in F_{T}(j)$, so $(i, u) \in S_{0}$. Since $i \neq h$ and $u \neq h$ we get $(i, u) \in S_{0}: h$.

Lemma 3.17. Let $U \subseteq C_{2, n}$ and $a \in[n]$. Then $I_{U: a}=I_{U}:\left(x_{a}\right)$.

Proof. $I_{U}=\bigcap_{\{i, j\} \in U}\left(x_{i}, x_{j}\right)$. Then

$$
I_{U}:\left(x_{a}\right)=\bigcap_{\{i, j\} \in U}\left(\left(x_{i}, x_{j}\right):\left(x_{a}\right)\right)=\bigcap_{\{i, j\} \in U: a}\left(x_{i}, x_{j}\right)=I_{U: a} .
$$

Lemma 3.18. Let $S=T \cup S_{0}$ be a generalized tower set. For every a $\in$ $\pi_{1}\left(S_{0}\right)$, there is $(a, h) \in S_{0}$ such that $I_{S: a}+\left(x_{h}\right)$ is a complete intersection ideal of height 2 .

Proof. We proceed step by step.

1) In this first step we show that the assertion is equivalent to prove that for all $\{i, j\} \in \varphi(S: a)$ we have either $\{i, h\} \in \varphi(S: a)$ or $\{j, h\} \in \varphi(S: a)$.

At the beginning we observe that if $I_{S: a}+\left(x_{h}\right)$ is equidimensional of height 2 and $\mathfrak{p}$ is a minimal prime in its primary decomposition then $x_{h} \in \mathfrak{p}$, so $I_{S: a}+\left(x_{h}\right)=\bigcap_{i}\left(x_{h}, x_{i}\right)=\left(x_{h}, \prod_{i} x_{i}\right)$ that is a complete intersection.

On the other hand to show that $I_{S: a}+\left(x_{h}\right)$ is equidimensional of height 2 it is enough to prove that for all $\{i, j\} \in \varphi(S: a)$ we have either $\{i, h\} \in \varphi(S: a)$ or $\{j, h\} \in \varphi(S: a)$. In fact let $\mathfrak{p}=$ $\left(x_{i}, x_{j}, x_{h}\right)$ be a prime ideal containing $I_{S: a}+\left(x_{h}\right)$, so $\{i, j\} \in \varphi(S$ : $a)$, consequently $\{i, h\} \in \varphi(S: a)$ or $\{j, h\} \in \varphi(S: a)$ i.e. $\mathfrak{p}$ contains a prime ideal of height 2 containing $I_{S: a}+\left(x_{h}\right)$.

Now let $a \in \pi_{1}\left(S_{0}\right)$, and let $m_{1}=\min S^{a}$; we set

$$
U\left(S^{a}\right):=\left\{m \in S^{a} \mid T_{m}=T_{m_{1}} \text { and } F_{T}(m)=\emptyset\right\} .
$$

Note that by Lemma 3.14, $m_{1} \in U\left(S^{a}\right)$.

We claim that the integer $h$ which we are looking for can be found in $U\left(S^{a}\right)$. In the next two steps we prove properties of $U\left(S^{a}\right)$ for our claim.

2) Let $m, n \in U\left(S^{a}\right)$ then $T^{m}=T^{n}$. If $\alpha \in T^{m} \backslash T^{n}$, i.e. $(m, \alpha) \in T$ and $(n, \alpha) \notin T$ we have $T_{\alpha} \supset T_{m}=T_{n}$. Now since $\alpha \notin F_{T}(n)=\emptyset$ and $(n, \alpha) \notin T$ we should have $T_{\alpha} \subseteq T_{n}$, a contradiction.

3) If $F_{T}(m)=\emptyset$ and $(\alpha, \beta) \in T$ then either $(\alpha, m) \in T$ or $(m, \beta) \in T$. Since $\beta \notin F_{T}(m)=\emptyset$ we get either $T_{\beta} \subseteq T_{m}$, hence $(\alpha, m) \in T$, or $(m, \beta) \in T$.

In the remaining steps we will find the integer $h$ working by induction on $\left|U\left(S^{a}\right)\right|$. 
4) If $\left|U\left(S^{a}\right)\right|=1$ then $U\left(S^{a}\right)=\left\{m_{1}\right\}$, and we would like to show that for all $\{i, j\} \in \varphi(S: a)$ we have either $\left\{i, m_{1}\right\} \in \varphi(S: a)$ or $\left\{j, m_{1}\right\} \in \varphi(S: a)$. If $\{i, j\} \in \varphi(T)$ then by item 3 we are done. So we can assume that $\{i, j\} \in \varphi\left(S_{0}\right)$, with $(i, j) \in S_{0}$. Of course we can suppose that $j \neq m_{1}$. Since $j \notin F_{T}\left(m_{1}\right)$ then either $\left(m_{1}, j\right) \in T$ or $T_{j} \subseteq T_{m_{1}}$. If $\left(m_{1}, j\right) \in T$ we are done; otherwise we can assume that $T_{j} \subseteq T_{m_{1}}$.

- if $T_{j} \subset T_{m_{1}}$ then either $\left(j, m_{1}\right) \in T$ and we are done, or $\left(j, m_{1}\right) \notin T$ then $m_{1} \in F_{T}(j)$, so by item 4 in Definition 3.12 we get $\left(i, m_{1}\right) \in S_{0}$ and we are done again.

- if $T_{m_{1}}=T_{j}$, let us suppose that

$$
\left\{i, m_{1}\right\},\left\{m_{1}, j\right\} \notin \varphi(S) .
$$

Since $S$ is connected then, taking $(i, j)$ and $\left(a, m_{1}\right)$, we get $(a, j) \in$ $S_{0}$. Since $U\left(S^{a}\right)=\left\{m_{1}\right\}$ and $j \neq m_{1}$ we have $F_{T}(j) \neq \emptyset$. Now take $k \in F_{T}(j)$; then $T_{m_{1}}=T_{j} \subset T_{k}$, so $k<m_{1}$. Since $(a, j) \in S_{0}$ and $k \in F_{T}(j)$ by item 4 in Definition 3.12, we have $(a, k) \in S_{0}$, and this contradicts the minimality of $m_{1}$.

Let now $U\left(S^{a}\right)=\left\{m_{1}, \ldots, m_{p}\right\}, p>1$.

5) At first we prove that $U\left(S^{a}\right) \backslash\left\{m_{p}\right\}=U\left(\left(S: m_{p}\right)^{a}\right)$. The inclusion $U\left(S^{a}\right) \backslash\left\{m_{p}\right\} \subseteq U\left(\left(S: m_{p}\right)^{a}\right)$ follows directly by definition of $U\left(S^{a}\right)$ and by Lemma 3.15. Let $m \in U\left(\left(S: m_{p}\right)^{a}\right)$ then $m \neq m_{p}$ and $\left(T: m_{p}\right)_{m}=\left(T: m_{p}\right)_{m_{1}}$; since $T_{m_{1}}=T_{m_{p}}$ we get $m_{p} \notin T_{m_{1}}$ therefore $T_{m} \supseteq T_{m_{1}}$. By the minimality of $m_{1}$ we have $T_{m}=T_{m_{1}}$. Now if $b \in F_{T}(m)$ then $T_{m_{1}}=T_{m} \subset T_{b}$, so $b<m_{1}$. Since $S$ is a generalized tower set we get $(a, b) \in S_{0}$ and this contradicts the minimality of $m_{1}$.

6) By the inductive hypothesis there exists $m \in U\left(S^{a}\right) \backslash\left\{m_{p}\right\}$ such that for any $(i, j) \in(S: a): m_{p}$ we have either

$$
\{m, i\} \text { or }\{m, j\} \in \varphi\left((S: a): m_{p}\right) .
$$

We will prove that either $m_{p}$ or $m$ is the wanted element. Let us suppose that there exist $(\alpha, \beta),(u, v) \in S$ such that

$\{m, \alpha\},\{m, \beta\} \notin \varphi((S: a))$ and $\left\{m_{p}, u\right\},\left\{m_{p}, v\right\} \notin \varphi((S: a))$.

Note that $\beta=m_{p}$, since otherwise $(\alpha, \beta) \in S: m_{p}$. Now since $v \neq m_{p}(u, v) \in S_{0}: m_{p}$, hence by hypothesis on $m$ it should be either $(u, m) \in S_{0}$ or $\{v, m\} \in \varphi(T)$; but the last assertion is false since, by item $2, T_{m}=T_{m_{p}}$ and $T^{m}=T^{m_{p}}$. This implies that $\left(\alpha, m_{p}\right)$ and $(u, m) \in S$ and this contradicts the connection of $S$.

Finally we are ready to prove the announced result.

Theorem 3.19. If $S$ is a generalized tower set then $S$ is aCM.

Proof. $S=T \cup S_{0}$, where $T$ is a tower set and for $S_{0}$ the properties of Definition 3.12 hold. We proceed by induction on $r=\left|\pi_{1}\left(S_{0}\right)\right|$. If $S_{0}=\emptyset$ then $S=T$ which is aCM by Theorem 2.6. Now we can suppose that the assertion is true up to $r-1$. Take $a \in \pi_{1}\left(S_{0}\right)$. Note that $S: a=T \cup\left(S_{0}: a\right)$ 
and $S: a$ is a generalized tower set with respect to this decomposition. Then by inductive hypothesis $S: a$ is aCM. We can write $I_{S}=I_{S: a} \cap\left(x_{a}, f\right)$ where $f=\prod_{j \in S^{a}} x_{j}$. Using the exact sequence

$$
0 \rightarrow I_{S} \rightarrow I_{S: a} \oplus\left(x_{a}, f\right) \rightarrow I_{S: a}+\left(x_{a}, f\right) \rightarrow 0
$$

it is enough to show that $\operatorname{proj-} \operatorname{dim}\left(I_{S: a}+\left(x_{a}, f\right)\right) \leq 3$, i.e. $\operatorname{proj-\operatorname {dim}}\left(I_{S: a}+\right.$ $(f)) \leq 2$. To do that we use induction on $\operatorname{deg} f=\left|S^{a}\right|$. If $f=x_{h}$ then, by Lemma 3.18, $I_{S: a}+\left(x_{h}\right)$ is a complete intersection ideal of height 2 and we are done.

If $\operatorname{deg} f=\left|S^{a}\right|>1$, by Lemma 3.18, there is $h \in S^{a}$ such that $I_{S: a}+\left(x_{h}\right)$ is a complete intersection ideal of height 2 . We can write

$$
I_{S: a}+(f)=\left(\left(I_{S: a}:\left(x_{h}\right)\right)+\left(f_{h}\right)\right) \cap\left(I_{S: a}+\left(x_{h}\right)\right),
$$

where $f_{h}=f / x_{h}$. In fact $I_{S: a}+(f) \subseteq\left(\left(I_{S: a}:\left(x_{h}\right)\right)+\left(f_{h}\right)\right) \cap\left(I_{S: a}+\left(x_{h}\right)\right)$ trivially. Let $g \in\left(\left(I_{S: a}:\left(x_{h}\right)\right)+\left(f_{h}\right)\right) \cap\left(I_{S: a}+\left(x_{h}\right)\right)$ be a monomial. If $g \in I_{S: a}$ we are done, otherwise $g \in\left(x_{h}\right)$. If $g \in\left(f_{h}\right)$ then $g \in(f)$ and we are done again. Otherwise $x_{h} g \in I_{S: a}$; since $I_{S: a}$ is a monomial squarefree ideal, we get $g \in I_{S: a}$.

By Lemma 3.17 we have that $I_{S: a}:\left(x_{h}\right)=I_{(S: h): a}$. Since by Lemma 3.16 $S: h$ is a generalized tower set, observing that $f_{h}=\prod_{j \in(S: h)^{a}} x_{j}$, we can apply the inductive hypothesis to assert that

$$
\operatorname{proj-dim}\left(\left(I_{S: a}:\left(x_{h}\right)\right)+\left(f_{h}\right)\right) \leq 2 .
$$

We set $J:=\left(I_{S: a}:\left(x_{h}\right)\right)+\left(f_{h}\right)$. Now let us consider the exact sequence

$$
0 \rightarrow I_{S: a}+(f) \rightarrow J \oplus\left(I_{S: a}+\left(x_{h}\right)\right) \rightarrow J+\left(x_{h}\right) \rightarrow 0 .
$$

Since $\operatorname{proj}-\operatorname{dim}\left(J+\left(x_{h}\right)\right) \leq 3$ and $I_{S: a}+\left(x_{h}\right)$ is a complete intersection ideal of height 2 , we can conclude that $\operatorname{proj}-\operatorname{dim}\left(I_{S: a}+(f)\right) \leq 2$.

Now we want to give a converse of the previous theorem. More precisely we want to prove that every monomial squarefree aCM ideal of height two is supported on a suitable generalized tower set. To do this we introduce some preparatory material.

Definition 3.20. Let $U \subseteq C_{2, n}$. We will say that $U$ is generalized towerizable set if there exists an ordinante function $\omega: C_{2, n} \rightarrow\left(\mathbb{Z}_{+}^{2}\right)^{*}$ and a permutation $\tau$ on $\pi_{2}(\omega(U))$ such that $\tau \omega(U)$ is a generalized tower set.

Let $U \subseteq C_{2, n}$ be an aCM set; then, by the Hilbert-Burch theorem, $I_{U}$ is a determinantal ideal generated by the maximal minors of a matrix of size $(r+1) \times r$.

Lemma 3.21. If $I \subset R$ is an aCM monomial ideal of height 2 then it admits a Hilbert-Burch matrix of the form

$$
\left(\begin{array}{ccccccccccc}
M_{0,1} & 0 & \ldots & 0 & & & & & & \ldots & 0 \\
D_{1} & M_{1,2} & \ldots & M_{1, \alpha_{1}} & 0 & \ldots & & & & & \ldots \\
0 & D_{2} & 0 & 0 & M_{2, \alpha_{1}+1} & \ldots & M_{2, \alpha_{2}} & M_{3, \alpha_{2}+1} & \ldots & M_{3, \alpha_{3}} & \ldots \\
& 0 & D_{3} & 0 & 0 & \ldots & 0 & & & & \\
& & & \ddots & & & & & & & \\
& & & & & & & & & & \\
& & & & & & & & & \ddots & \\
& & & & & & & & \ldots & 0 & D_{r}
\end{array}\right)
$$


where $D_{i}$ and $M_{i j}$ are monomials, $D_{i} \neq 0$ and $M_{i j} \neq 0$ and $D_{i}$ is in the position $(i, i)$ (we enumerate the rows from 0 to $r$ and the columns from 1 to $r$ ).

Proof. We take the minimal monomial set $G$ of generators for $I$, then the first syzygy module is minimally generated by a set $\Phi$ of $r$ elements acting each only on two of such generators. Moreover there are at least two generators in $G$ on which only one syzygy acts. Let $f_{0}$ be one of these generators and let $\phi_{1}$ be the syzygy acting on $f_{0}$ and let $f_{1}$ be the other generator on which acts $\phi_{1}$. Now we call $\phi_{2}, \ldots, \phi_{\alpha_{1}}$ all the other syzygies in $\Phi$ acting respectively on $f_{1}$ and $f_{2}, \ldots, f_{\alpha_{1}} \in G$. By iterating this procedure we get our matrix.

Definition 3.22. An Hilbert-Burch matrix of the type as in Lemma 3.21 will be called a matrix of standard form.

Definition 3.23. Let $\mathcal{M}=\left(m_{i j}\right)$ be an Hilbert-Burch matrix of standard form of size $(r+1) \times r$. Let $\sigma:[r] \rightarrow\{0, \ldots, r-1\}$ be the application such that $\sigma(j)$ is the only integer less than $j$ such that $m_{\sigma(j) j} \neq 0$.

From now on we set $M_{i}$ for $M_{\sigma(i), i}$.

Remark 3.24. Note that $\sigma(1)=0, \sigma(2)=1$ and, for $j>2, \sigma(j) \geq \sigma(j-1)>$ 0 .

Given $j \in\{1, \ldots, r\}$ we denote with $m(j)$ the set

$$
m(j):=\left\{j, \sigma(j), \sigma^{2}(j), \ldots, \sigma^{h}(j)\right\},
$$

where $h$ is the only integer such that $\sigma^{h}(j)=1$. We write $u \notin m(j)$ to mean $u \in[n] \backslash m(j)$.

Remark 3.25. Note that if $i \in m(j)$ then $m(i) \subseteq m(j)$.

We denote by $f_{i}$ the determinant of the matrix obtained by removing the row $i$ for $0 \leq i \leq r$. By the Hilbert-Burch theorem we have that

$$
\left\{f_{0}, \ldots, f_{r}\right\}
$$

is a minimal set of generators for $I$. Note that $f_{0}=D_{0} \cdots D_{r}$. In the following proposition will compute all the other generators.

Proposition 3.26. For any $i \in\{1, \ldots, r\}$, with the above notation, we have

$$
f_{i}=\prod_{j \in m(i)} M_{j} \cdot \prod_{j \notin m(i)} D_{j} .
$$

Proof. Let $i \in\{1, \ldots, r\}$, and let $H$ be the square matrix given by $\mathcal{M}$ without the row containing $D_{i}$. Since $M_{i}$ is the only entry in the $i$-th column of $H$, we compute the determinant $f_{i}$ by using the Laplace expansion along its $i$-th column. Thus $f_{i}=M_{i} G_{1}$, where $G_{1}$ is the determinant of the matrix $H_{1}$ obtained from $H$ by deleting the row $\sigma(i)$ and the $i$-th column. Note that $M_{\sigma(i)}$ is the only entry in the $\sigma(i)$-th column of $H_{1}$, hence $f_{i}=M_{i} M_{\sigma(i)} G_{2}$, where $G_{2}$ is the determinant of the matrix $H_{2}$ obtained from $H_{1}$ by deleting the row $\sigma^{2}(i)$ and the $\sigma(i)$-th column. So, by iterating this computation, we get $f_{i}=\prod_{j \in m(i)} M_{j} \cdot G^{\prime}$, where $G^{\prime}$ is the determinant of the matrix $H^{\prime}$ obtained from $H$ by deleting the rows $\sigma(j)$ and the columns $j$, for all $j \in$ $m(i)$. Finally, we observe that $H^{\prime}$ is an upper triangular matrix, therefore $G^{\prime}=\prod_{j \notin m(i)} D_{j}$ 
Now we want to construct a generalized tower set starting from a $n$ Hilbert-Burch matrix of standard form $\mathcal{M}$.

Definition 3.27. We define

$$
U_{\mathcal{M}}^{\prime}:=\left\{\{i, j\} \in C_{2,2 r} \mid i<j \leq r \text { and } i \notin m(j)\right\}
$$

and

$$
U_{\mathcal{M}}^{\prime \prime}:=\left\{\{i, j\} \in C_{2,2 r} \mid i \leq r<j \text { and } j-r \in m(i)\right\} .
$$

Finally we set

$$
U_{\mathcal{M}}:=U_{\mathcal{M}}^{\prime} \cup U_{\mathcal{M}}^{\prime \prime}
$$

Proposition 3.28. 1) If $\{u, v\} \in U_{\mathcal{M}}^{\prime}$ then either $u \notin m(i)$ or $v \notin m(i)$ for every $1 \leq i \leq r$.

2) If $\{u, v\} \in U_{\mathcal{M}}^{\prime \prime}$, with $u<v$, then either $u \notin m(i)$ or $v-r \in m(i)$ for every $1 \leq i \leq r$.

Proof. 1) Let $u, v \in m(i)$ with $u<v$. Then $v=\sigma^{h}(i)$ and $u=\sigma^{k}(i)$ with $h<k$. Then $u=\sigma^{k-h} \sigma^{h}(i)=\sigma^{k-h}(v)$, i.e. $u \in m(v)$, hence $\{u, v\} \notin U_{\mathcal{M}}^{\prime}$.

2) Let $\{u, v\} \in U_{\mathcal{M}}^{\prime \prime}$, with $u<v$, such that $u \in m(i)$. Since $v-r \in m(u)$ we get $v-r \in m(i)$.

Proposition 3.29. $U_{\mathcal{M}}$ is connected.

Proof. Let $\{i, j\},\{u, v\} \in U_{\mathcal{M}}$, with $i<j, u<v$ and $u<i$. If $u \notin m(i)$ then $u<i \leq r$, so $\{u, i\} \in U_{\mathcal{M}}^{\prime}$. If $u \in m(i)$, we have to consider two cases.

If $v \leq r$ then $\{u, v\} \in U_{\mathcal{M}}^{\prime}$, hence $u \notin m(v)$. Since $u \in m(i)$ we get $m(i) \nsubseteq m(v)$ i.e. $i \notin m(v)$. Moreover, by Proposition 3.28 we have $v \notin m(i)$ and so $\{i, v\} \in U_{\mathcal{M}}^{\prime}$.

If $v>r$ then, by Proposition 3.28, we get $v-r \in m(i)$, so $\{i, v\} \in U_{\mathcal{M}}^{\prime \prime}$.

Now we set $\mathcal{T}:=U_{\mathcal{M}}^{\prime} \cup\left\{\{i, j\} \in U_{\mathcal{M}}^{\prime \prime} \mid j-r \in m(r)\right\}$ and $\mathcal{S}_{0}:=U_{\mathcal{M}} \backslash \mathcal{T}$, so $\mathcal{S}_{0}=\left\{\{i, j\} \in C_{2,2 r} \mid i \leq r<j\right.$ and $\left.j-r \in m(i) \backslash m(r)\right\}$.

For every $i \in[r]$, we set $\mu_{i}:=\max (m(i) \cap m(r))$.

We set

$$
\mathcal{V}_{i}^{(1)}:=\left\{\{u, v\} \in U_{\mathcal{M}}^{\prime} \mid \mu_{u}=\mu_{v}=\mu_{i}\right\}
$$

and

$$
r_{i}^{(1)}:=\max \bigcup_{\alpha \in \mathcal{V}_{i}^{(1)}} \alpha \cup\{0\} .
$$

Note that $r_{i}^{(1)}<r$. Moreover, we set

$$
\mu_{i}^{(1)}:=\left\{\begin{array}{ll}
\max \left(m(i) \cap m\left(r_{i}^{(1)}\right)\right) & \text { if } r_{i}^{(1)}>0 \\
0 & \text { otherwise }
\end{array} .\right.
$$

Now by induction let us suppose that we defined $\mu_{i}^{(k)}$ for $1 \leq k<h$. Then we set

$$
\mathcal{V}_{i}^{(h)}:=\left\{\{u, v\} \in \mathcal{V}_{i}^{(h-1)} \mid \mu_{u}^{(h-1)}=\mu_{v}^{(h-1)}=\mu_{i}^{(h-1)}\right\}
$$

and

$$
r_{i}^{(h)}:=\max \bigcup_{\alpha \in \mathcal{V}_{i}^{(h)}} \alpha \cup\{0\} .
$$


Note that if $r_{i}^{(h-1)}>0$ then $r_{i}^{(h)}<r_{i}^{(h-1)}$. If $r_{i}^{(h-1)}=0$ then $r_{i}^{(h)}=0$. Moreover we set

$$
\mu_{i}^{(h)}:=\left\{\begin{array}{ll}
\max \left(m(i) \cap m\left(r_{i}^{(h)}\right)\right) & \text { if } r_{i}^{(h)}>0 \\
0 & \text { otherwise }
\end{array} .\right.
$$

In the sequel we will set $\mu_{i}^{(0)}:=\mu_{i}$. Note that by definition the sequence $\left(\mu_{i}^{(h)}\right)_{h \geq 0}$ vanishes definitively.

Lemma 3.30. If $\{i, j\} \in U_{\mathcal{M}}^{\prime}$ then there exists $h$ such that $\mu_{i}^{(h)} \neq \mu_{j}^{(h)}$.

Proof. If $\mu_{i}^{(h)}=\mu_{j}^{(h)}$ for every $h$ then $\mathcal{V}_{i}^{(h)}=\mathcal{V}_{j}^{(h)} \neq \emptyset$ for every $h$. Consequently $\left(\mu_{i}^{(h)}\right)_{h \geq 0}$ should not be definitively null, a contradiction.

For every $\{i, j\} \in U_{\mathcal{M}}^{\prime \prime}$, with $i>j$, we set $\omega(\{i, j\}):=(i, j)$.

For every $\{i, j\} \in U_{\mathcal{M}}^{\prime}$, let $t$ be the smallest integer such that $\mu_{i}^{(t)} \neq \mu_{j}^{(t)}$ (see Lemma 3.30). Then we set

$$
\omega(\{i, j\}):=\left\{\begin{array}{l}
(i, j) \text { if } \mu_{i}^{(t)}<\mu_{j}^{(t)} \\
(j, i) \text { if } \mu_{i}^{(t)}>\mu_{j}^{(t)}
\end{array} .\right.
$$

Now we set $\bar{U}:=\omega\left(U_{\mathcal{M}}\right) \subset\left(\mathbb{Z}_{+}^{2}\right)^{*}$.

Observe that for $1 \leq i \leq r,(r+1, i) \in \bar{U}$, hence $\pi_{2}(\bar{U})=[r]$.

Lemma 3.31. Let $i, j \in[r]$, with $\mu_{i} \leq \mu_{j}$. Let $h>r$ be such that $(h, i) \in$ $\omega(\mathcal{T})$. Then $(h, j) \in \omega(\mathcal{T})$.

Proof. Since $\{h, i\} \in \mathcal{T}$ and $h>r$ we get $h-r \in m(i) \cap m(r)$. On the other hand, $\mu_{i} \leq \mu_{j}$ implies that $m(i) \cap m(r) \subseteq m(j) \cap m(r)$. Therefore $h-r \in m(j) \cap m(r)$, so $(h, j) \in \omega(\mathcal{T})$.

Theorem 3.32. $U_{\mathcal{M}}$ is a generalized towerizable set.

Proof. Let us consider $\bar{U}$. It is enough to find a permutation $\tau$ on $[2 r]$ such that $\tau(\bar{U})$ is a generalized tower set.

We set $\bar{T}:=\omega(\mathcal{T})$. We want to show that the set $\left\{\bar{T}_{i}\right\}_{1 \leq i \leq r}$ is totally ordered by inclusion. Let $i, j \in[r], i \neq j$.

Case $1: \mu_{i}^{(k)}=\mu_{j}^{(k)}$ for every $k \geq 0$. In this case we will show that $\bar{T}_{i}=\bar{T}_{j}$. Of course it is enough to show that $\bar{T}_{i} \subseteq \bar{T}_{j}$. Take $h \in \bar{T}_{i}$ i.e. $(h, i) \in \bar{T}$. If $h>r$ by Lemma $3.31(h, j) \in \bar{T}$. If $h \leq r$ let $t$ be the smallest integer such that $\mu_{h}^{(t)}<\mu_{i}^{(t)}=\mu_{j}^{(t)}$. By the minimality of $t, r_{i}^{(t)}=r_{h}^{(t)}$, hence from $m(h) \cap m\left(r_{h}^{(t)}\right) \subset m(i) \cap m\left(r_{i}^{(t)}\right)=m(j) \cap m\left(r_{j}^{(t)}\right)$ it follows that $j \notin m(h)$. On the other hand if $h \in m(j)$, since $\mu_{i}^{(k)}=\mu_{j}^{(k)}$ for every $k \geq 0$, by Lemma 3.30, $\{i, j\} \notin U_{\mathcal{M}}^{\prime}$ i.e. either $i \in m(j)$ or $j \in m(i)$. If $j \in m(i)$ then $h \in m(i)$ and this contradicts that $\{h, i\} \in \mathcal{T}$. If $i \in m(j)$, since both $h$ and $i$ belong to $m(j)$ then either $h \in m(i)$ or $i \in m(h)$, again a contradiction with $\{h, i\} \in \mathcal{T}$. Therefore $h \notin m(j)$. This together with $j \notin m(h)$, as we saw, implies that $\{h, j\} \in \mathcal{T}$. By the inequality $\mu_{h}^{(t)}<\mu_{j}^{(t)}$, we get $(h, j) \in \bar{T}$.

Case 2 : let $t$ be the smallest integer such that $\mu_{i}^{(t)}<\mu_{j}^{(t)}$. We claim that $\bar{T}_{i} \subseteq \bar{T}_{j}$. Take $h \in \bar{T}_{i}$ i.e. $(h, i) \in \bar{T}$. If $h>r$, using again Lemma 3.31, we 
are done. So we can assume $h \leq r$. Let $s$ be the smallest integer such that $\mu_{h}^{(s)}<\mu_{i}^{(s)}$. Assume $s \leq t$ (the same argument will work in the case $s>t$ ). We have to prove that $(h, j) \in \bar{T}$. From the inequalities $\mu_{h}^{(s)}<\mu_{i}^{(s)} \leq \mu_{j}^{(s)}$ it is enough to show that $\{h, j\} \in \mathcal{T}$ i.e. $h \notin m(j)$ and $j \notin m(h)$. Using the same inequalities we get

$$
m(h) \cap m\left(r_{h}^{(s)}\right) \subset m(i) \cap m\left(r_{i}^{(s)}\right) \subseteq m(j) \cap m\left(r_{j}^{(s)}\right) .
$$

As above, by the minimality of $s$, we have $r_{h}^{(s)}=r_{i}^{(s)}=r_{j}^{(s)}$, so we can deduce that $j \notin m(h)$. On the other hand, if $h \in m(j)$, since there is $k \in m(j) \cap m\left(r_{j}^{(s)}\right)$, with $k \notin m(h)$, we obtain that $h \in m(k)$ (note that we are using the fact that $h, k \in m(j)$ and $k \notin m(h))$. Since $m(k) \subseteq m(j) \cap m\left(r_{j}^{(s)}\right)$, we get $h \in m\left(r_{j}^{(s)}\right)$, so $h \in m(i)$, a contradiction, hence $h \notin m(j)$ and we are done.

Let $\tau$ be a permutation on $[2 r]$, such that $\tau(i)<\tau(j)$ whenever $1 \leq i, j \leq r$ and $\bar{T}_{i} \supseteq \bar{T}_{j}$. Moreover $\tau(i)=i$ for $i \geq r+1$. We denote by $S=\tau \omega\left(U_{\mathcal{M}}\right)$. We want to show that $S$ is a generalized tower set i.e. we need to prove the four properties stated in Definition 3.12,

1) Clearly $S$ is connected by Lemma 3.29 .

2) Now set $T:=\tau(\bar{T})$ and $S_{0}=S \backslash T$. By the properties of $\tau, T$ is a tower set.

3) Note that $\pi_{2}(S)=\pi_{2}(T)=[r]$ and $\pi_{1}(T) \subseteq[r] \cup\{r+1 \leq i \leq 2 r \mid$ $i-r \in m(r)\}$. Therefore if $(i, j) \in S_{0},\left\{\tau^{-1}(i), \tau^{-1}(j)\right\} \in U_{\mathcal{M}}^{\prime \prime}$. This implies that $i>r$ so $i \notin \pi_{2}(T)$. On the other hand since $\left\{\tau^{-1}(i), \tau^{-1}(j)\right\} \notin \mathcal{T}$, we have $i-r \notin m(r)$ i.e. $i \notin \pi_{1}(T)$. Let $u=\tau^{-1}(j)$. Then $(i, u) \notin \bar{T}$, so $\{i, u\} \in U_{\mathcal{M}}^{\prime \prime}$. We claim that $(u, r) \in \bar{T}$, which will imply that $j \in \pi_{1}(T)$. We need to show that $u \notin m(r)$. We know that $\{i, u\} \in U_{\mathcal{M}}^{\prime \prime}$, so $i-r \in$ $m(u)$. If $u \in m(r)$ then $i-r \in m(r)$ therefore $(i, j) \in T$, a contradiction. Consequently $j \in \pi_{1}(T) \cap \pi_{2}(T)$.

4) Now we would like to prove that if $(i, \tau(j)) \in S_{0}$ and $\tau(u) \in F_{T}(\tau(j))$ then $(i, \tau(u)) \in S_{0}$, i.e. $\{i, u\} \in \mathcal{S}_{0}$ hence we have to show that $i-r \in m(u)$ and $i-r \notin m(r)$. Since $(i, \tau(j)) \in S_{0}$ we have $i-r \in m(j) \backslash m(r)$. Since $\tau(u) \in F_{T}(\tau(j))$ we have $T_{\tau(j)} \subset T_{\tau(u)}$ and $(\tau(j), \tau(u)) \notin T$. From this we deduce that $\{u, j\} \notin U_{\mathcal{M}}^{\prime}$, consequently either $j \in m(u)$ or $u \in m(j)$. If $j \in m(u)$ since $i-r \in m(j)$ we get $i-r \in m(u)$ and we are done. If $u \in m(j)$ since also $i-r \in m(j)$ we get either $u \in m(i-r)$ or $i-r \in m(u)$. If $u \in m(i-r)$ and $i-r \notin m(u)$, take $v \in \bar{T}_{u} \backslash \bar{T}_{j}$ and consider $\{u, v\}$, $\{i, j\} \in U_{\mathcal{M}}$. Since $U_{\mathcal{M}}$ is connected we have that one of the following sets must belong to $U_{\mathcal{M}}$ :

$$
\{u, i\},\{u, j\},\{v, i\},\{v, j\} .
$$

Note that $\{u, i\} \notin U_{\mathcal{M}}$ since $i-r \notin m(u)$. Moreover $\{u, j\} \notin U_{\mathcal{M}}$ (see above). If $\{v, i\} \in U_{\mathcal{M}}$, then $(i, v) \in \omega\left(\mathcal{S}_{0}\right)$, so $i-r \in m(v)$, consequently $u \in m(v)$, which contradicts that $\{u, v\} \in U_{\mathcal{M}}$.

If $\{v, j\} \in U_{\mathcal{M}}$, then $(j, v) \in \omega\left(\mathcal{S}_{0}\right)$. Therefore $j \in \bar{T}_{v} \backslash \bar{T}_{u}$ and $v \in \bar{T}_{u} \backslash \bar{T}_{v}$, a contradiction since $\bar{T}_{u}$ and $\bar{T}_{v}$ are comparable by inclusion. Consequently $i-r \in m(u)$ and we are done. 
Remark 3.33. We note that if a Hilbert-Burch matrix of standard form $\mathcal{M}=\left(m_{i, j}\right)$ is bidiagonal (i.e. the only non zero entries are $m_{i, i}$ and $m_{i, i+1}$ ) then $U_{\mathcal{M}}$ is a towerizable set. This follows by Theorem 3.32, since, using the same notation as above, $\mathcal{S}_{0}=\emptyset$. Vice versa if $U_{\mathcal{M}}$ is a towerizable set then it is easy to build a bidiagonal Hilbert-Burch matrix of standard form $\mathcal{M}^{\prime}$ such that $I(\mathcal{M})=I\left(\mathcal{M}^{\prime}\right)$. This generalizes a result of Ahn and Shin in [AS].

In order to get our main result we need the following Lemma.

Lemma 3.34. With the same terminology as above, let $\mathcal{M}$ be a HilbertBurch matrix of standard form. Let $\{i, j\},\{u, v\}$ be two different elements in $U_{\mathcal{M}}$. Then, if we set $H_{i}:=D_{i}$ and $H_{r+i}:=M_{i}$ for $i=1, . ., r$,

$$
\operatorname{depth}\left(H_{i}, H_{j}, H_{u}, H_{v}\right) \geq 3 .
$$

Proof. We need to distinguish three possibilities. If $\{i, j\},\{u, v\} \in U_{\mathcal{M}}^{\prime}$ then $i, j, u, v \leq r$, since $\prod_{h=1 . . r} D_{h}$ is a minimal generator of $I(\mathcal{M})$ and $|\{i, j, u, v\}| \geq 3$ we are done. If $\{i, j\},\{u, v\} \in U_{\mathcal{M}}^{\prime \prime}$, say $i, u \geq r$, then if $j=v$ we have $i-r \in m(j)$ and $u-r \in m(j)$. So, by Proposition [3.26, $H_{i} H_{u}$ is a factor of $f_{j}$ hence $H_{i}, H_{u}$ are coprime as $f_{j}$ is squarefree, then $\operatorname{depth}\left(H_{i}, H_{j}, H_{u}\right)=3$. If $j<v$, then $i-r \in m(j)$ and $v \notin m(j)$. So, by Proposition 3.26, $H_{i} H_{v}$ is a factor of $f_{j}$ hence $H_{i}, H_{v}$ are coprime as $f_{j}$ is squarefree, then $\operatorname{depth}\left(H_{i}, H_{j}, H_{v}\right)=3$. If $\{i, j\} \in U_{\mathcal{M}}^{\prime},\{u, v\} \in U_{\mathcal{M}}^{\prime \prime}$, with $v<u$, when $|\{i, j, v\}|=3$ we are done, otherwise say $v=j$ then $H_{u} H_{i}$ is a factor of $f_{j} \operatorname{so} \operatorname{depth}\left(H_{i}, H_{j}, H_{u}\right)=3$.

Collecting all the results of this section we are ready to proof the main result.

Theorem 3.35. Let $I \subset k\left[x_{1}, \ldots, x_{n}\right]$ be a monomial squarefree of height 2. Then I is aCM iff it defines a generalized tower scheme.

Proof. Let us suppose that $I$ is aCM. Then $I=I(\mathcal{M})$ for some $\mathcal{M}$ of standard form of size $(r+1) \times r$ (see Lemma 3.21 and Definition 3.22). By Theorem $3.32 U_{\mathcal{M}}$ is a generalized towerizable set. Let $\omega$ and $\tau$ be as in the proof of Theorem 3.32, Let $S:=\tau\left(\omega\left(U_{\mathcal{M}}\right)\right)$, which is a generalized tower set. For $j \in \pi_{1}(S)$, we set

$$
f_{1 j}:=\left\{\begin{array}{ll}
D_{\tau^{-1}(j)} & \text { for } j \leq r \\
M_{j-r} & \text { for } j>r
\end{array} .\right.
$$

For $j \in \pi_{2}(S)=[r]$, we set $f_{2 j}:=D_{\tau^{-1}(j)}$. Also we set

$$
\mathcal{F}_{i}:=\left\{f_{i j} \mid j \in \pi_{i}(S)\right\}, i=1,2 .
$$

By Lemma 3.34, $\mathcal{F}_{1}$ and $\mathcal{F}_{2}$ satisfy the conditions of genericity required by Definition 3.13 .

We claim that $I_{S}\left(\mathcal{F}_{1}, \mathcal{F}_{2}\right)=I$. At first we show that $I \subseteq I_{S}\left(\mathcal{F}_{1}, \mathcal{F}_{2}\right)$. Indeed, let $g_{k}$ be the maximal minor obtained from $\mathcal{M}$ by deleting the $k$ th row and take any $(i, j) \in S$. We need to show that $g_{k} \in\left(f_{1 i}, f_{2 j}\right)$ for every $k$. Since $g_{0}=\prod_{1 \leq i \leq r} D_{i}$ and $f_{2 j}=D_{\tau^{-1}(j)}, g_{0} \in\left(f_{1 i}, f_{2 j}\right)$. Assume $k \geq 1$. Since $(i, j) \in S$ we have $\left\{\tau^{-1}(i), \tau^{-1}(j)\right\} \in U_{\mathcal{M}}=U_{\mathcal{M}}^{\prime} \cup U_{\mathcal{M}}^{\prime \prime}$. If 
$\left\{\tau^{-1}(i), \tau^{-1}(j)\right\} \in U_{\mathcal{M}}^{\prime}$ then $\left(f_{1 i}, f_{2 j}\right)=\left(D_{\tau^{-1}(i)}, D_{\tau^{-1}(j)}\right)$ and by Proposition 3.28 we get either $\tau^{-1}(i) \notin m(k)$ or $\tau^{-1}(j) \notin m(k)$ therefore by Proposition 3.26, $g_{k} \in\left(f_{1 i}, f_{2 j}\right)$. We proceed analogously if $\left\{\tau^{-1}(i), \tau^{-1}(j)\right\} \in U_{\mathcal{M}}^{\prime \prime}$.

Now we show that $I_{S}\left(\mathcal{F}_{1}, \mathcal{F}_{2}\right) \subseteq I$. Let $g \in I_{S}\left(\mathcal{F}_{1}, \mathcal{F}_{2}\right)$ be a squarefree monomial. Let

$$
E_{g}=\{0\} \cup\left\{1 \leq h \leq r \mid g \in\left(M_{h}\right) \text { and } g \notin\left(D_{h}\right)\right\} .
$$

We set $e:=\max E_{g}$. We claim that $g \in\left(g_{e}\right)$.

Assume $e=0$ and let $h \in[r]$. If $g \in\left(M_{h}\right)$ then $g \in\left(D_{h}\right)$ by the maximality of $e$. If $g \notin\left(M_{h}\right)$ then $g \in\left(D_{h}\right)$ since $\{r+h, h\} \in U_{\mathcal{M}}^{\prime \prime}$.

Assume $e>0$. Remind that $g_{e}=\prod_{j \in m(e)} M_{j} \cdot \prod_{j \notin m(e)} D_{j}$. If $g \in\left(M_{h}\right)$ then $g \in\left(D_{h}\right)$ for $h>e$ by the maximality of $e$. If $g \notin\left(M_{h}\right)$ then $g \in\left(D_{h}\right)$ since $\{r+h, h\} \in U_{\mathcal{M}}^{\prime \prime}$. Note that if $e=1$ we are done. So we can assume $e>1$. If $h \notin m(e)$ and $h<e$ then $\{e, h\} \in U_{\mathcal{M}}^{\prime}$, consequently $g \in\left(D_{e}, D_{h}\right)$, but $g \notin\left(D_{e}\right)$ so $g \in\left(D_{h}\right)$. Now let $h \in m(e)$, namely $\{r+h, e\} \in U_{\mathcal{M}}^{\prime \prime}$. Therefore $g \in\left(M_{h}, D_{e}\right)$, but $g \notin\left(D_{e}\right)$ so $g \in\left(M_{h}\right)$. Since $g_{e}$ is a squarefree monomial, we showed that $g \in\left(g_{e}\right)$.

Vice versa let us suppose that $I$ defines a generalized tower scheme. This means that $I=I_{S}\left(\mathcal{F}_{1}, \mathcal{F}_{2}\right)$ where $S$ is a generalized tower set and $\mathcal{F}_{1}, \mathcal{F}_{2}$ are families of monomials, satisfying the conditions of Definition 3.13. By Theorem 3.19, $S$ is aCM. So we get that $I$ is aCM just applying Proposition 3.7.

\section{REFERENCES}

[AS] J. Ahn, and Y. S. Shin. The minimal free resolution of a star-configuration in $\mathbb{P}^{n}$ and the Weak Lefschetz Property. J. Korean Math. Soc 49.2 (2012): 405-417.

[GHM] A. V. Geramita, B. Harbourne, and J. Migliore. Star configurations in $\mathbb{P}^{n}$. J. of Algebra 376 (2013): 279-299.

[GHS] A. V. Geramita, T.Y. Harima, S. Shin, Extremal Point Sets and Gorenstein Ideals. Adv. in Math. 152(1) (2000), 78-119.

[GS] A. V. Geramita, S. Shin, k-configurations in $\mathbb{P}^{3}$ all have extremal resolutions. J. of Algebra 213 (1999): 351-368.

[MR] R. Maggioni, A. Ragusa, Connections between Hilbert function and geometric properties for a finite set of points in $\mathbb{P}^{2}$, Le Matematiche, 39 (1984), no. 1-2, $153-170$.

[RZ] A. Ragusa, G. Zappalà, Partial intersection and graded Betti numbers, Beiträge zur Algebra und Geometrie, 44 (2003), no. 1, 285-302.

(G. Favacchio) Dip. di Matematica e Informatica, Università di Catania,

Viale A. Doria 6, 95125 Catania, Italy

E-mail address: favacchio@dmi.unict.it

Fax number: +39095330094

(A. Ragusa) Dip. di Matematica e Informatica, Università di Catania,

Viale A. Doria 6, 95125 Catania, Italy

E-mail address: ragusa@dmi.unict.it

Fax number: +39095330094

(G. Zappalà) Dip. di Matematica e Informatica, Università di Catania, Viale A. Doria 6, 95125 Catania, Italy 
E-mail address: zappalag@dmi.unict.it

Fax number: +39095330094 